\title{
DISEÑO DE UN MODELO DE ARQUITECTURA EMPRESARIAL PARA EL MACRO PROCESO DE GESTIÓN ACADÉMICA DE LA UNIVERSIDAD DE PAMPLONA
}

\author{
DESIGN OF AN ENTERPRISE ARCHITECTURE MODEL FOR BUSINESS \\ ACADEMIC PROCESS MANAGEMENT OF UNIVERSIDAD DE PAMPLONA
}

\author{
MSc. William Mauricio Rojas Contreras, MSc. Maritza del Pilar Sánchez Delgado. \\ Ing.(c) William Guillermo Guerrero Silva.
}

Universidad de Pamplona, Facultad de Ingenierías y Arquitectura, Grupo CICOM. Ciudadela Universitaria, Pamplona, Norte de Santander, Tel. (+577) 568 5303, Ext. 163. E-mail: \{mrojas, pilas\}@ unipamplona.edu.co, guillermoguerrero1226@gmail.com

Resumen: En este artículo se presenta el diseño de un modelo de Arquitectura Empresarial para el macro proceso de gestión académica de la Universidad de Pamplona donde se observa la integración entre el negocio, los sistemas de información y la infraestructura tecnológica que soporta a este macro proceso. Dicho modelo se elaboró adaptando y utilizando el Framework TOGAF para este caso en particular.

Palabras clave: Arquitectura empresarial, procesos de negocio, gestión académica, sistemas de información, framework, TOGAF.

\begin{abstract}
This paper describes the design of a model of Enterprise Architecture for the academic macro process management at the University of Pamplona where integration between the businesses is observed, information systems and technology infrastructure that supports this macro process is presented. This model was developed by adapting and using the TOGAF framework for this case.
\end{abstract}

Keywords: Enterprise architecture, business process, academic Management, information systems, framework, TOGAF.

\section{INTRODUCCIÓN}

En este artículo se presenta en primera parte una breve explicación del término de Arquitectura Empresarial (AE) y los dominios que esta comprende. A continuación se presenta una breve explicación del Framework TOGAF y como se adaptó para el diseño de este modelo. Seguidamente y de manera general se expone un diagnóstico de la arquitectura empresarial actual del macro proceso de gestión académica de la Universidad de Pamplona en sus cuatro dominios: el dominio de negocio, de datos, de aplicaciones y tecnológico. Posteriormente se presenta el diseño de esta Arquitectura Empresarial.
El auge de las tecnologías de información en la actualidad es tal que diariamente las organizaciones a nivel mundial se enfrentan a los constantes cambios que se presentan en las mencionadas tecnologías, produciendo cambios en el negocio de las empresas. La utilización de las tecnologías de información también ha aumentado de manera considerable en estas últimas décadas. Hoy en día la gran mayoría de organizaciones hacen uso de estas tecnologías como soporte de sus procesos y gestión de la información. Es importante para las organizaciones precisar una correcta integración entre el negocio y las tecnologías de información. Una falta de integración impulsa a las organizaciones a buscar 
una solución a dicho problema, una de estas soluciones es la Arquitectura Empresarial. La AE es "una metodología de mejora continua a mediano plazo, que basada en una visión integral, permite mantener actualizada la estructura de información organizacional alineando procesos, datos, aplicaciones e infraestructura tecnológica en cuatro dimensiones: negocios, datos/información, aplicaciones y tecnología” (Amazing, 2015).

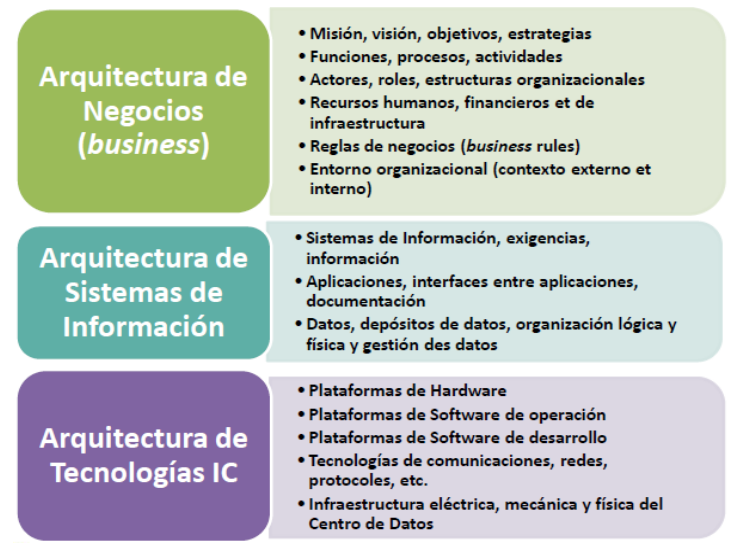

Fig. 1. Dominios de la Arquitectura Empresarial.

(Barrios Albornoz, ULA, \& UFPS, 2014)

En la Figura 1 se pueden observar los elementos que componen en cada dominio a la $\mathrm{AE}$, estos dominios representan un conjunto de arquitecturas que integran la arquitectura final. La arquitectura del Negocio representa el primer dominio y en esta se encuentra todo lo relacionado con la gobernabilidad, estrategias, misión, visión y el negocio como tal de la organización. La Arquitectura de Sistemas de Información comprende los datos y las aplicaciones que son usadas como apoyo en la realización de los procesos de la organización. El último dominio está representado por la arquitectura tecnológica, esta incorpora las soluciones tecnológicas para la comunicación, almacenamiento y gestión de la información y el manejo de servidores de datos y de aplicaciones.

Para la implementación de la práctica de la Arquitectura Empresarial, cada organización debe iniciar un proyecto que permita alcanzar los objetivos planteados que compensen las necesidades que dieron origen al desarrollo de esta práctica. Para una correcta implementación de una AE se deben llevar a cabo una serie de pasos que permitan una precisa ejecución de la misma. Estos pasos los puede plantear cada empresa o también se pueden guiar por metodologías ya creadas que ofrecen soluciones para un adecuado desarrollo de una AE. Es acá donde adquiere importancia el Framework de Arquitectura Empresarial.

Un Framework de AE "es una herramienta que se puede utilizar para el desarrollo de una amplia gama de diferentes arquitecturas. Debe describir un método para diseñar un sistema de información en términos de un conjunto de bloques de construcción, y cómo los bloques de construcción encajan. Debe contener un conjunto de herramientas y proporcionar un vocabulario común. También debe incluir una lista de estándares recomendados y los productos de cumplimiento que pueden ser utilizados para poner en práctica los elementos básicos" (The Open Group, 2006).

Entre los diferentes Frameworks de AE encontramos a TOGAF, The TOGAF Framework es tal vez el marco de referencia de Arquitectura Empresarial más utilizado hoy en día, gracias a su nivel de adaptación, al método de desarrollo de arquitectura genérico que posee y a la disponibilidad de información que ofrece. TOGAF es un marco - un método detallado y un conjunto de herramientas de apoyo - para el desarrollo de una arquitectura empresarial. Puede ser utilizado libremente por cualquier organización que desee desarrollar una arquitectura de la empresa para su uso dentro de dicha organización (The Open Group, 2013).

TOGAF se puede usar también con otros Frameworks de Arquitectura Empresarial, el núcleo de TOGAF es su Método de Desarrollo de Arquitectura o ADM por sus siglas en inglés (Architecture Development Method) que describe como se debe implementar la arquitectura empresarial dentro de la organización. TOGAF cubre cuatro tipos de arquitectura: la arquitectura de negocios, la arquitectura de datos, la arquitectura de aplicaciones y la arquitectura tecnológica.

La Figura 2 describe la estructura del Marco de Referencia TOGAF que se divide en siete partes, cada uno de estas es accedida a medida que se avanza por el Método de Desarrollo de Arquitectura o ADM pos sus siglas en inglés.

TOGAF es un Framework con gran capacidad de adaptabilidad en el desarrollo de AE en distintas organizaciones, su ADM que es el núcleo de funcionamiento del Framework describe las fases que se deben llevar a cabo para una correcta implementación de una Arquitectura Empresarial. 


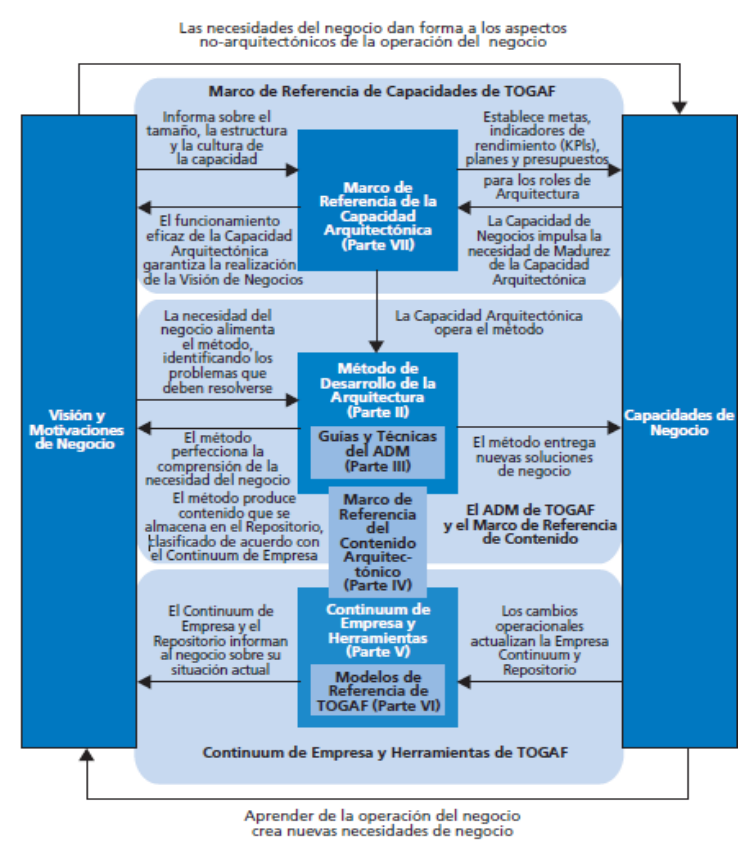

Fig. 2. Estructura de TOGAF.

Fuente: (The Open Group, 2013).

En la figura 3 se pueden observar las fases que comprende el ADM.

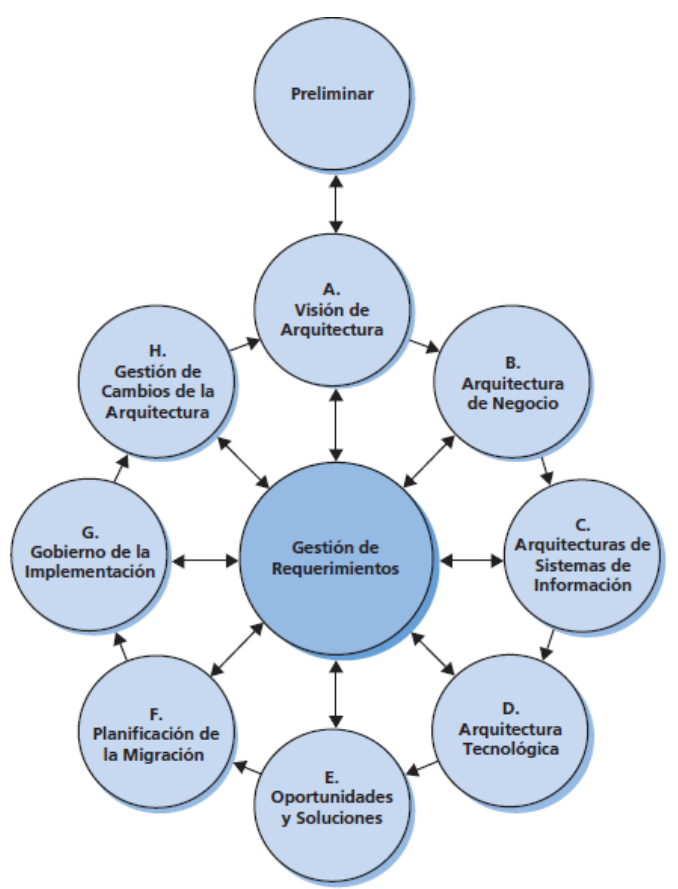

Fig. 3. Fases del ADM.

Fuente: (The Open Group, 2013)

Para el diseño del modelo de AE del macro proceso de gestión académica de la Universidad de Pamplona se utilizó el ADM en sus cuatro primeras fases.

\section{DIAGNÓSTICO DEL MACRO PROCESO DE GESTIÓN ACADÉMICA}

El diagnóstico se desarrolló en cada dominio de la AE del macro proceso de gestión académica, comenzando por el dominio del negocio, seguido del dominio de datos y de aplicaciones y por último el dominio de la infraestructura tecnológica.

\subsection{Arquitectura del Negocio}

La Universidad de Pamplona es una Institución de Educación Superior con tres macro procesos misionales: academia, investigación e interacción social. La Universidad tiene establecido el Sistema Integrado de Gestión, el cual contempla los procesos que se llevan a cabo en la Institución. El macro proceso de Gestión Académica, abordan los procesos necesarios desde la planificación académica hasta la autoevaluación de los programas. Cada proceso es detallado a nivel de procedimientos y actividades con responsables, registros e indicadores para su medición, entre otros.

\subsubsection{Lista de Procesos Documentados en el SIG}

- Planificación de la gestión académica.

- Selección Docente tiempo completo y medio tiempo.

- Responsabilidad académica.

- Docencia.

- Evaluación y desarrollo docente.

- Proceso para el trabajo de grado.

- Admisión registro y control académico pregrado.

- Admisión registro y control académico modalidad distancia.

- Solicitud o renovación registro calificado.

- Autoevaluación.

\subsection{Arquitectura de Datos}

La información asociada al macro proceso de Gestión Académica de la Universidad Pamplona es manejada a través de diferentes gestores de base de datos, en donde cada uno maneja la información utilizada por las distintas aplicaciones que apoyan el mencionado proceso.

La información asociada a los procesos administrativos y académicos dentro del macro proceso de Gestión Académica es manejada y almacenada por el Sistema Gestor de Base de Datos (SGBD) Oracle y PostgreSQL. 


\subsection{Arquitectura de Aplicaciones}

La Universidad de Pamplona apoya sus procesos de negocio haciendo uso de la suite Academusoft, que integra un conjunto de aplicaciones y módulos para la realización de las actividades académicas, administrativas y financieras logrando eficiencia y eficacia a la hora de alcanzar los objetivos del macro proceso de Gestión Académica, haciendo uso de la tecnología de información que se encuentran disponibles actualmente y en constante evolución. Estas aplicaciones son desarrolladas por el Centro de Investigación Aplicada y Desarrollo en Tecnologías de Información (CIADTI), además de desarrollarlas el CIADTI brinda soporte técnico y el servicio de infraestructura tecnológica para dichas aplicaciones. "Academusoft es una EAS (Enterprise Aplications Solutions) para las IES, que ofrece una alternativa de alto nivel para el ingreso, organización, gestión y administración de la información en cada uno de los procesos Académicos y necesidades de negocio generadas por la Institución Educativa, busca optimizar la generación de la información y apoyar a la alta gerencia en la toma de decisiones." (Plataforma, 2015)

Academusoft integra tres módulos que abarcan en su totalidad todas las funcionalidades que son utilizadas por los diferentes procesos y actividades en la gestión académica.

- Hermesoft - Campus Colaborativo

- Academusoft - Campus Académico

- Gestasoft - Campus Administrativo

\subsection{Arquitectura Tecnológica}

La infraestructura tecnológica del macro proceso de gestión académica de la Universidad de Pamplona soporta las aplicaciones y servidores que son utilizados en éste entorno y permite la comunicaciones entre ellas, además brinda seguridad frente a posibles ataques informáticos que se puedan presentar dentro o fuera de la organización.

El uso de la arquitectura de tres niveles, además de ser un estándar en internet garantiza que la comunicación se realice entre el usuario y el servidor de aplicación, brindando un nivel alto de seguridad frente a los datos.

La red local utilizada se basa en la topología estrella extendida en la cual se conectan la totalidad de los equipos a través de un cableado de fibra óptica centralizado.

\section{DISEÑO DEL MODELO DE ARQUITECTURA EMPRESARIAL}

El diseño del modelo de arquitectura empresarial para el macro proceso de gestión académica de la Universidad de Pamplona se basó en el marco de referencia TOGAF, que basa su funcionamiento en el método ADM el cual se divide en 9 fases:

- Fase preliminar

- Fase A: Visión de la arquitectura.

- Fase B: Arquitectura de negocio

- Fase C: Arquitectura de sistemas de información

- Fase D: Arquitectura Tecnológica

- Fase E : Oportunidades y soluciones

- Fase F: Planificación de la migración

- Fase G: Gobierno de la implementación

- Fase H: Gestión de cambios de la arquitectura.

Las fases de la $\mathrm{A}$ a la $\mathrm{H}$ son iterativas y siempre deben responder o estar alineadas con la gestión de los requerimientos. Para el diseño del modelo actual se tuvo en cuenta la fase preliminar y las fases A, B, C y D propuestas por el ADM.

\subsection{Fase preliminar}

En esta se definieron las actividades de preparación e iniciación de la arquitectura empresarial la definición del marco de referencia con el cual se va a desarrollar la arquitectura empresarial y la definición de principios.

El principal objetivo de la fase preliminar es determinar la capacidad arquitectónica deseada por la organización. Para llevar a cabo este objetivo fue necesario realizar una serie de pasos que describe TOGAF:

1. Determinar las organizaciones de la empresa que serán afectadas

2. Definir el alcance de la arquitectura empresarial.

3. Examinar el contexto organizacional para llevar a cabo Arquitectura Empresarial

4. Identificar y establecer los principios de arquitectura.

\subsection{FASE A: Visión de la Arquitectura.}

En esta fase se definió lo que está dentro y fuera del alcance del desarrollo de la arquitectura y las restricciones que se deben manejar además de identificar a los interesados o stakeholders. 


\subsubsection{Stakeholders}

- Consejo Superior

- Consejo Académico

- Rector

- Vicerrector Académico

- Decanos

- Directores de Departamento

- Directores de Programa

- Vicerrector Financiero y Administrativo

- Planeación

- Registro y Control Académico

- Usuarios:

Internos: docentes y administrativos

Externos: estudiantes, MEN, CNA, SPADIES, SUE

\subsection{FASE B: Arquitectura de Negocio.}

En esta fase se abordó lo referente a la arquitectura del negocio del macro proceso de gestión académica de la Universidad de Pamplona teniendo como base la arquitectura actual, los diferentes procesos de negocio que se llevan a cabo dentro de este macro proceso, qué actores intervienen y qué roles son asignados para cada actor.

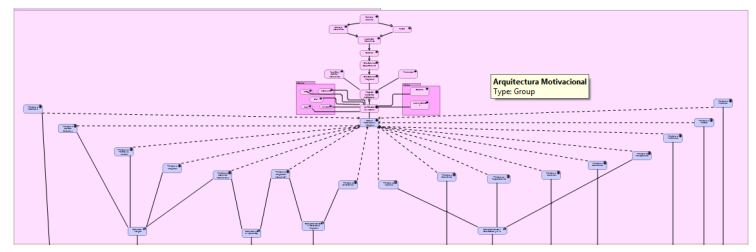

Fig. 4. Arquitectura Motivacional.

Dentro del dominio del negocio se encuentra la vista motivacional donde se observan los stakeholders o interesados, los clientes tanto internos y externos, los principios y los requerimientos fijados en el PEI que motivan al macro proceso de gestión académica en la Universidad de Pamplona.

En la Figura 5 se expone la arquitectura del negocio del macro proceso de gestión académica, aquí se detallan cada uno de los servicios ofrecidos, y los procesos encargados de su realización.

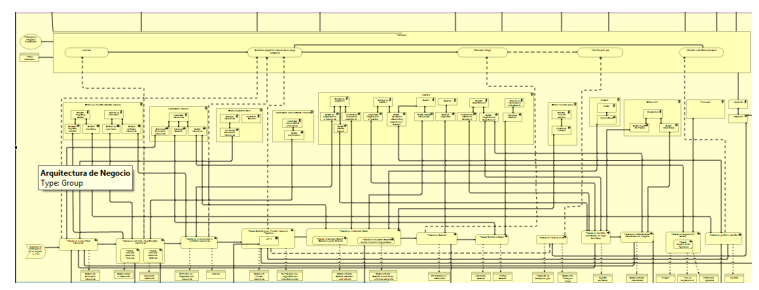

Fig. 5. Arquitectura de negocio.
Dentro de la arquitectura del negocio también se describen una serie de actores con unos roles definidos dentro de una localización, los roles se asignan a uno o varios procesos dentro del dominio. Cada actor puede tener uno o más roles dependiendo del proceso en donde este actue. Cada proceso produce una documentación que se puede observar en la vista del negocio.

\subsection{FASE C: Arquitectura de Sistemas de Información.}

La arquitectura de sistemas de información se divide en dos vistas, la vista de datos y la vista de aplicaciones. La vista de datos no se presenta por motivos de confidencialidad.

\subsubsection{Arquitectura de Aplicaciones.}

Dentro de esta arquitectura se detallan las aplicaciones que apoyan el macro proceso de gestión académica de la Universidad de Pamplona.

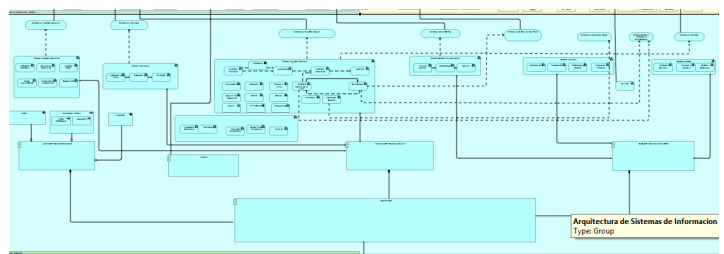

Fig. 6. Arquitectura de Aplicaciones.

En la arquitectura de aplicaciones se observan en la primera parte los servicios de aplicaciones que ayudan en la realización de los procesos de la gestión académica, estos servicios son realizados por una serie de funcionalidades encapsuladas en módulos, cada módulo está contenido en un componente de aplicación ya sea Hermesoft Campus Colaborativo, Academusoft - Campus Académico o Gestasoft - Campus Administrativo. Estos tres componentes de aplicación constituyen la suite Academusoft.

\subsection{FASE D: Arquitectura Tecnológica.}

La fase D es la cuarta fase del ciclo ADM de TOGAF, esta fase describe la arquitectura tecnológica, lo referente a servidores, seguridad y conexiones que soportan y dan apoyo a los procesos dentro del macro proceso de gestión académica de la Universidad de Pamplona. 


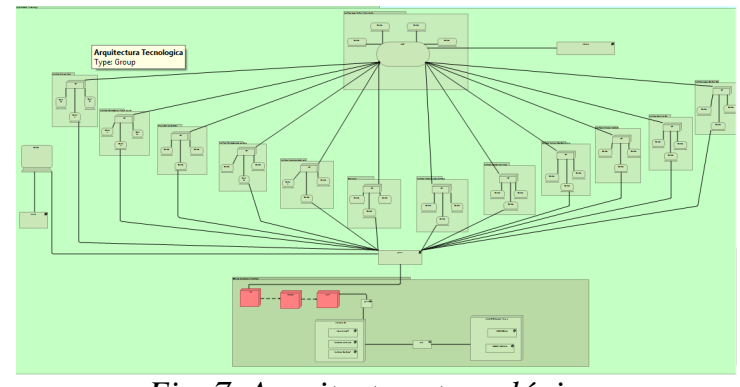

Fig. 7. Arquitectura tecnológica.

La Figura 7 ilustra la arquitectura tecnológica que soporta y da apoyo al macro proceso de gestión académica de la Universidad de Pamplona, en esta se puede observar la topología de red utilizada y la seguridad empleada para la prevención de ataques o intrusiones informáticas por terceras personas ajenas al sistema.

\section{CONCLUSIONES}

Con base en los fundamentos de Arquitectura Empresarial y sus respectivos Frameworks se ha desarrollado el diseño del Modelo de Arquitectura Empresarial para el macro proceso de Gestión Académica de la Universidad de Pamplona el cual proporciona una nueva forma o manera de ver el macro proceso mencionado donde se puede hacer un mejor control del proceso así como una mejor evolución y cambio del mismo.

Se ha comprobado la capacidad de adaptabilidad de TOGAF para los proyectos de Arquitectura Empresarial en las instituciones educativas logrando así adaptarlo a un proceso inmerso en este tipo de organizaciones como lo es el de gestión académica.

El dominio del negocio del macro proceso de Gestión Académica de la Universidad de Pamplona es una vista fuerte dentro de la práctica de Arquitectura Empresarial ya que todos los procesos del negocio se encuentran documentados en el Sistema Integrado de Gestión

A través del desarrollo de este trabajo se deduce que la práctica de Arquitectura Empresarial se puede implementar en las organizaciones educativas y la gran mayoría de organizaciones en el país no han iniciado un proyecto de este carácter gran parte por la falta de información sobre el tema y las metodologías que permiten un correcto desarrollo de esta.

\section{RECONOCIMIENTO}

Un reconocimiento especial al equipo del CIADTI por sus aportes respecto a sistemas de información e infraestructura tecnológica de la Universidad de Pamplona.

\section{REFERENCIAS}

Arango Serna, M. D., Londoño Salazar, J. E., \& Zapata Cortes, J. A. (2010). Arquitectura Empresarial - Una visión general. Revista Ingenierías Universidad de Medellín, 101 - 111.

Barrios Albornoz, J., ULA, \& UFPS. (Septiembre de 2014). Conferencia sobre Arquitectura Empresarial (AE) y el enfoque TOGAFF. Cúcuta, Colombia.

The Open Group. (2006). TOGAF Version 8 Enterprise Edition: What is an Architectura Framework? Ed. Berkshire.

The Open Group. (2013). Standard TOGAF Version 9.1. Guía de Bolsillo. Reino Unido: Van Haren Publishing.

Whittle, R., \& Myrick, C. (2004). Enterprise Business Architecture: The Formal link between strategy and results. USA: Boca Ratón.

\section{SITIOS WEB}

Amazing. (2015) Arquitectura Empresarial. http://www.amazing.com.co/arquitecturaempresarial.php (consultado: 03/03/2015)

Plataforma (2015). Universidad de Pamplona. http://plataforma.unipamplona.edu.co/ (consultado: 03/06/2015) 